\title{
High mobility polymer gated organic field effect transistor using zinc phthalocyanine
}

\author{
K R RAJESH*, V KANNAN, M R KIM, Y S CHAE and J K RHEE \\ Millimeter- Wave Innovation Technology Research Centre (MINT), Dongguk University, \\ Seoul 100-715, Republic of Korea
}

MS received 15 October 2012; revised 25 April 2013

\begin{abstract}
Organic thin film transistors were fabricated using evaporated zinc phthalocyanine as the active layer. Parylene film prepared by chemical vapour deposition was used as the organic gate insulator. The annealing of the samples was performed at $120{ }^{\circ} \mathrm{C}$ for $3 \mathrm{~h}$. At room temperature, these transistors exhibit $p$-type conductivity with field-effect mobilities ranging from $0.025-0.037 \mathrm{~cm}^{2} / \mathrm{Vs}$ and a $\left(I_{\mathrm{on}} / I_{\mathrm{off}}\right)$ ratio of $\sim 10^{3}$. The effect of annealing on transistor characteristics is discussed.
\end{abstract}

Keywords. Organic semiconductor; field effect transistor; phthalocyanine; high mobility.

\section{Introduction}

Organic field effect transistors (OFETs) are being extensively studied for various applications of electronic circuits (Horowitz 1998; Dimitrakopoulos and Malenfant 2002) including radiofrequency identification (RFID) tags, smart cards, digital paper displays and back planes for flexible active-matrix organic light-emitting diodes (AMOLEDs). The device performances of OFETs have improved dramatically over the past decade. The carrier mobility of OFETs is similar to or even higher than that of typically obtained ones with hydrogenated amorphous silicon TFTs, which have found widespread use in liquid crystal displays (AMLCDs) (Dimitrakopoulos and Malenfant 2002; Sundar et al 2004).

Phthalocyanines have been in the limelight due to their promising applications as organic semiconductors. Among them, zinc phthalocyanine $(\mathrm{ZnPc})$ is one of the most important, because of its broad spectral dependence. The basic properties of many metal phthalocyanines have been studied in detail by various researchers (Gould 1985; Belgachi and Collins 1998). They also exhibit strong non-linear optical properties, due to their spatially extended $\pi$-electron system (Fang et al 1993). In order to utilize the mechanical flexibility inherently possessed by organic materials, many attempts have been made to develop polymeric gate dielectric layers. Recently, a significant amount of effort has been made to deposit organic semiconductors onto polymeric insulators with the ultimate goal of creating all-organic transistors to power flexible display elements (Halik et al 2002). The performance characteristics of OFETs, such as their operating voltage, depends critically on the gate dielectric materials and their interfacial properties, because the dielectric surface

\footnotetext{
*Author for correspondence (rajthinfilms@yahoo.co.in, rajesh@dongguk.edu)
}

is in contact with the channel of OFETs, where the drain current flows (Veres et al 2004; Chua et al 2005). The interaction between these surfaces and the deposited organic can have a profound effect on thin film growth and the resulting electrical characteristics, since most of the charge transport in these structures occurs near the organic-insulator interface.

We used parylene- $\mathrm{C}$ as the gate insulator, which can be deposited by chemical vapour deposition (CVD) on the surface of ITO coated glass at room temperature and can form a parylene/organic interface with a low density of electronic defects (Gershenson et al 2006). Parylene forms pin hole free, thin conformal transparent coatings with excellent dielectric and mechanical properties (Podzorov et al 2003). It is used to passivate the entire area of the substrate except for ITO contact areas. The application of parylene CVD process to realize $\mathrm{ZnPc}$ OFETs has not been reported before. We determined the transistor parameters of the device at room temperature. Comparison of electrical properties of the virgin and annealed devices provides insight into the trap distributions.

\section{Experimental}

OFETs using $\mathrm{ZnPc}$ with a top contact structure were fabricated on ITO coated glass substrates. The cross-sectional structures and bias configurations of OFETs are schematically shown in figure 1 . ITO was selectively etched to form electrical contact for the gate insulator. Thoroughly cleaned etched ITO glass substrates with a surface sheet resistance of $10 \Omega / \square$ were used as the substrates.

The gate dielectric was a $490 \mathrm{~nm}$ thick parylene-C layer deposited in a lab coater. The dimer para-xylylene was vapourized in the vapourization zone at $\sim 135{ }^{\circ} \mathrm{C}$, cleaved in the pyrolysis zone at $\sim 695{ }^{\circ} \mathrm{C}$, and polymerized in the 


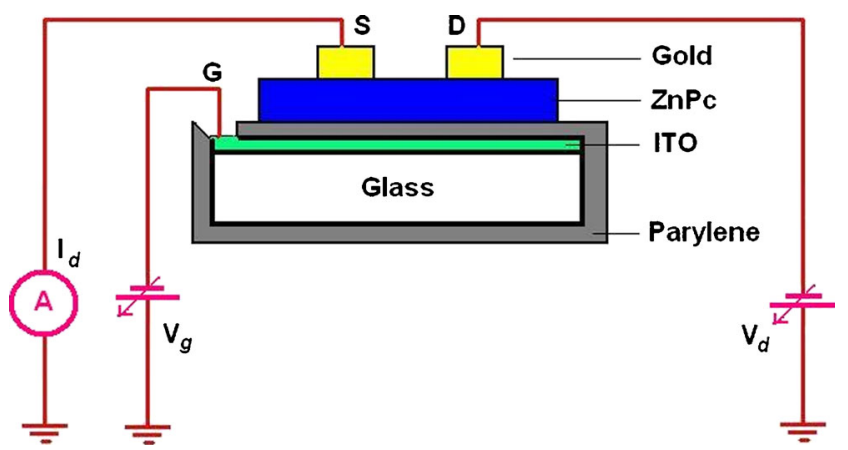

Figure 1. Schematic of OFETs. Gate is negatively biased to operate in accumulation mode.

deposition zone (the chamber), at room temperature and a pressure of $\sim 0.1$ Torr.

ZnPc powder (Aldrich Inc.) was evaporated onto the parylene films kept at room temperature using a Hanvac UHV coating plant at a pressure of $10^{-7}$ Torr. The evaporation rate was kept at $0 \cdot 1-0.2 \mathrm{~nm}$ per s and the thickness was $\sim 100 \mathrm{~nm}$. Finally, source/drain gold contacts with a thickness of $60 \mathrm{~nm}$ were evaporated through a shadow mask. The channel length and width-to-length channel ratio were, respectively, $50 \mu \mathrm{m}$ and 20.

Half of the samples were annealed at $120^{\circ} \mathrm{C}$ for $3 \mathrm{~h}$ in a furnace attached to a programmable temperature controller. The electrical characterizations of OFETs were carried out at room temperature using an Agilent E5272 semiconductor parameter analyser interfaced with a probe station kept in the dark. The capacitance of the parylene film was measured with HP 4294 LCR meter. The atomic force microscope (AFM) images of the samples were recorded by a digital instruments nanoscope.

\section{Results and discussion}

The fabricated OFETs operate in accumulation mode. The typical transistor characteristics are measured at room temperature in air immediately after device fabrication. Here, we measure the drain current $I_{\mathrm{d}}$ of the as-prepared FETs as a function of the drain voltage $V_{\mathrm{d}}$, as shown in figure 2 .

The voltage is varied from 0 to $-80 \mathrm{~V}$ in steps of $20 \mathrm{~V}$. The sharp increase of the drain current, $I_{\mathrm{d}}$, at negative values of the gate voltage, $V_{\mathrm{g}}$, indicates the formation of $p$-type conducting channel. Figure 3 shows corresponding transfer curves of the same device, $V_{\mathrm{g}}$ is varied from 20 to $-100 \mathrm{~V}$ with the application of $V_{\mathrm{d}}=-70 \mathrm{~V}$.

The field-effect mobility $\mu$ and threshold voltage $V_{\mathrm{t}}$ are calculated using (2)

$$
I_{\mathrm{d}, \mathrm{sat}}=\left(\frac{W}{2 L}\right) C_{\mathrm{i}} \mu\left(V_{\mathrm{g}}-V_{\mathrm{t}}\right)^{2},
$$

where $I_{\mathrm{d} \text {,sat }}$ is the drain current in the saturation region, $C_{\mathrm{i}}$ the gate capacitance per unit area and $V_{\mathrm{g}}$ the applied gate

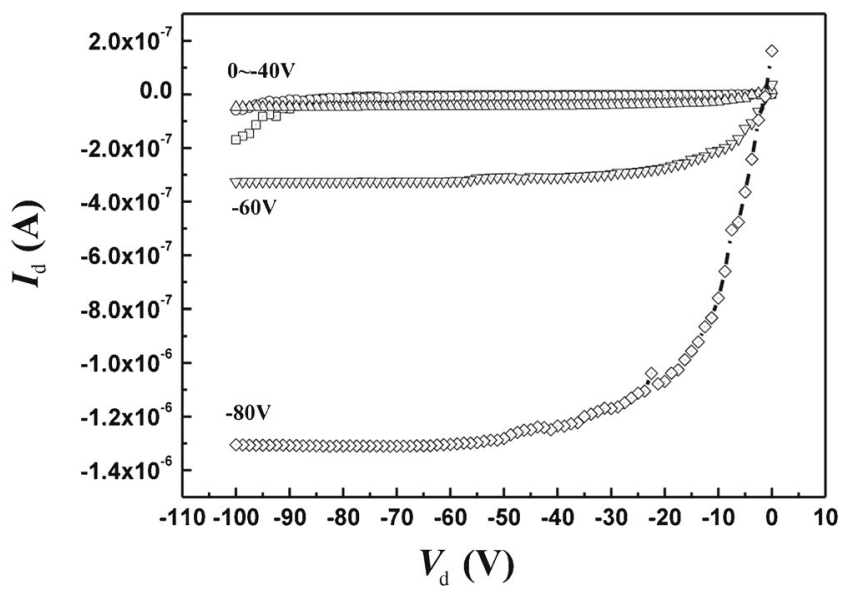

Figure 2. Output characteristics of as-deposited OFETs.

voltage. Using (1) and the values, $W$ (width of the channel) $=$ $1000 \mu \mathrm{m}, L$ (length of the channel) $=50 \mu \mathrm{m}$ and $C_{\mathrm{i}}=$ $7.2 \mathrm{nF} / \mathrm{cm}^{2}$, we obtain $\mu=0.012 \mathrm{~cm}^{2} / \mathrm{Vs}$ and $V_{\mathrm{t}}=-34.7 \mathrm{~V}$ at $V_{\mathrm{d}}=-40 \mathrm{~V}$. The maximum on/off current ratio $\left(I_{\mathrm{on}} / I_{\mathrm{off}}\right)$ is $8.64 \times 10^{2}$. The sub-threshold swing (SS) is defined as the gate voltage $\left(V_{\mathrm{g}}\right)$ required to modulate the drain current $\left(I_{\mathrm{d}}\right)$ by one decade as given by

$$
\mathrm{SS}=\ln 10 \frac{d V_{\mathrm{g}}}{d\left(\ln I_{\mathrm{d}}\right)} .
$$

The value of SS is calculated to be $15.29 \mathrm{~V} / \mathrm{dec}$. In practice, SS increases with increasing density of trap states at the semiconductor/insulator interface. Also at $V_{\mathrm{d}}=-70 \mathrm{~V}$, $\mu=0.025 \mathrm{~cm}^{2} / \mathrm{Vs}$ and $V_{\mathrm{t}}=-47.6 \mathrm{~V}$. In this case, on/off current ratio is $1.32 \times 10^{3}$ and $\mathrm{SS}$ is $18.95 \mathrm{~V} / \mathrm{dec}$. This large value of $V_{\mathrm{t}}$ is due to the trapping of accumulated holes. In pentacene, it has been reported that water molecules are localized between the molecules of pentacene and create trap states in the bandgap (Tsetseris and Pantelides 2007), and that the orientation of the water dipoles changes the local polarization of the organic monolayers, creating trap states in the bandgap (Pernstich et al 2005). The switch-on voltage $\left(V_{\text {so }}\right)$ corresponding to the flat band potential (Meijer et al 2002) obtained from figure 3 is found to be +0.51 V. Indeed, a positive onset voltage has been reported in some top-gated OFETs (Ling et al 2006). This effect is attributed to the doping of $\mathrm{ZnPc}$ with oxygen or moisture. Oxygen was previously shown to create deep acceptor levels and to increase the residual doping level of the organic semiconductor, both of which contribute to the positive shift of the onset voltage (Knipp et al 2006).

Figures 4 and 5 show TFT characteristics of the annealed samples. Using the same procedure, we obtain $\mu=$ $0.02 \mathrm{~cm}^{2} / \mathrm{Vs}$ and $V_{\mathrm{t}}=32.06 \mathrm{~V}$ at $V_{\mathrm{d}}=-40 \mathrm{~V} . I_{\mathrm{on}} / I_{\text {off }}$ ratio and $\mathrm{SS}$ are $3.15 \times 10^{3}$ and $12.87 \mathrm{~V} / \mathrm{dec}$, respectively. At $V_{\mathrm{d}}=-70 \mathrm{~V}$, the values for $\mu$ and $V_{\mathrm{t}}$ are $0.037 \mathrm{~cm}^{2} / \mathrm{Vs}$ and $42.56 \mathrm{~V}$, respectively. The corresponding $I_{\mathrm{on}} / I_{\mathrm{off}}$ ratio 


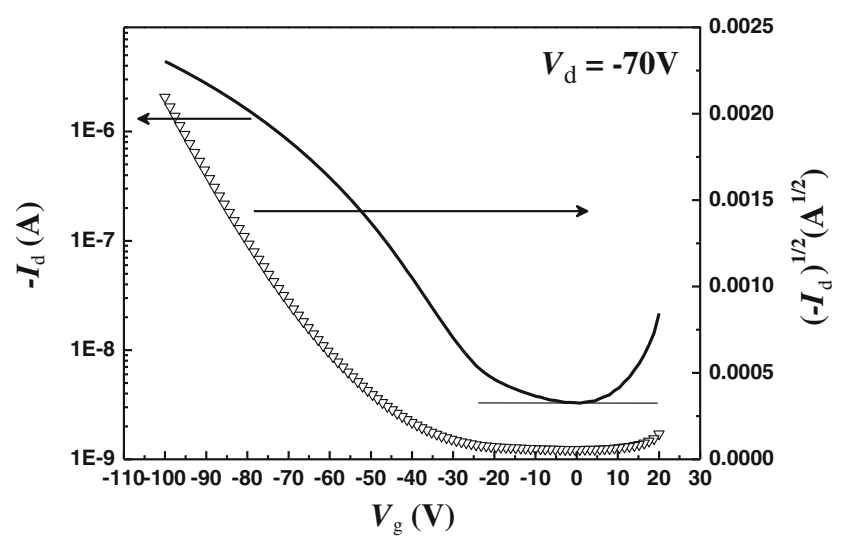

Figure 3. Transfer characteristics of as-deposited OFETs. Drain voltage, $V_{\mathrm{d}}=70 \mathrm{~V}$.

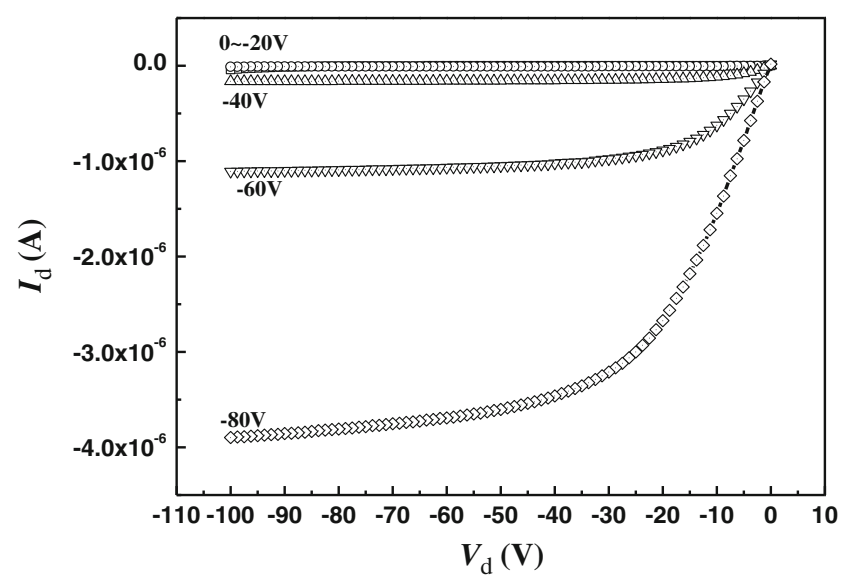

Figure 4. Output characteristics of OFETs annealed at $120^{\circ} \mathrm{C}$ for $3 \mathrm{~h}$.

and SS are $6.94 \times 10^{3}$ and $14.50 \mathrm{~V} / \mathrm{dec}$, respectively. In organic semiconductors, in disordered form, mobility will be a function of the applied field (Brïtting 2005). The change in mobility with voltage can be attributed to this. The mobility obtained for $\mathrm{ZnPc}$ is the highest among the various phthalocyanines in disordered form. ZnPc with a mobility of $0.32 \mathrm{~cm}^{2} / \mathrm{Vs}$ has been cited (Liqiang Li et al 2008). In this work an ultra-thin buffer layer (para-hexaphenyl) has been utilized between the insulator and semiconductor (Wang et al 2007). This structure is slightly different from the present work. The switch-on voltage $V_{\text {so }}$ obtained from figure 5 is found to be $-4.8 \mathrm{~V}$. This lowering of $V_{\mathrm{so}}$ indicates that the annealing process results in the removal of considerable quantities of oxygen acceptor impurities from the sample. Comparing the as-deposited and annealed samples, a decrease in the threshold voltage of the latter can be observed and this can be attributed to the injection improvement at the interface.

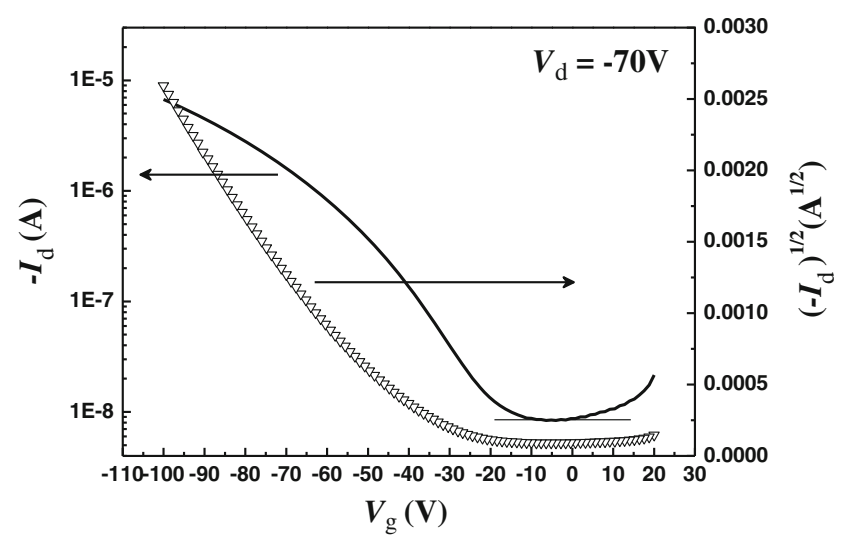

Figure 5. Transfer characteristics of OFETs annealed at $120^{\circ} \mathrm{C}$ for $3 \mathrm{~h}$. Drain voltage, $V_{\mathrm{d}}=70 \mathrm{~V}$.

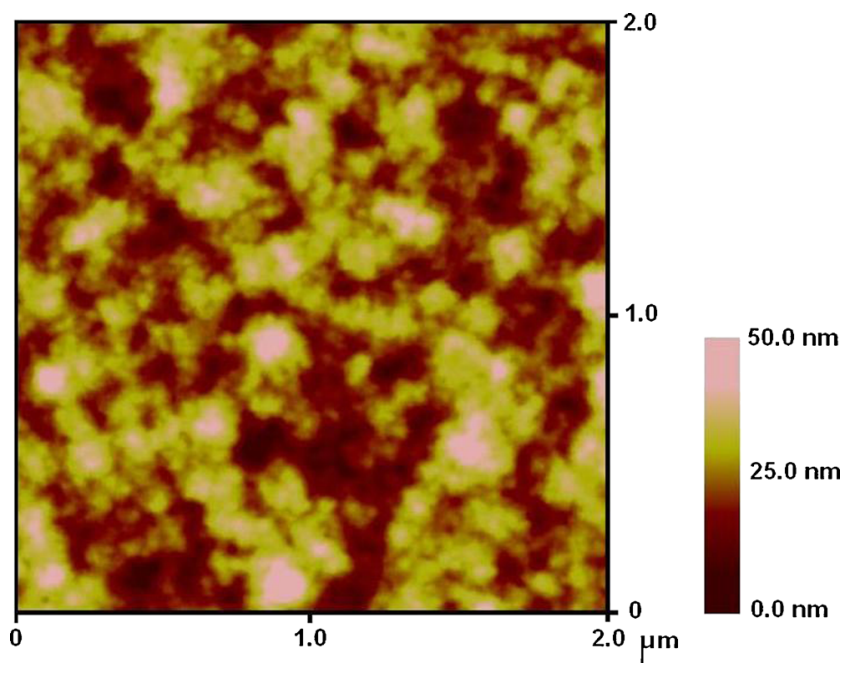

Figure 6. AFM topograph of parylene film deposited on ITO substrate.

Further analysis is possible by finding the interface parameter, $N_{\mathrm{ss}}^{\max }$. This corresponds to the maximum number of interface traps present and can be calculated using (3), assuming that the densities of the deep bulk states and interface states are independent of the energy (Rolland et al 1993; Unni et al 2005):

$$
N_{\mathrm{ss}}^{\max }=\left[\frac{s s \cdot \log (s)}{k T / q}-1\right] \frac{c_{i}}{q},
$$

where $k$ is the Boltzmann's constant, $T$ the absolute temperature and $q$ the electronic charge. The values of $N_{\mathrm{ss}}^{\max }$ calculated for the as-deposited $\mathrm{ZnPc}$ and annealed devices are $9.91 \times 10^{12} \mathrm{~cm}^{-2} \mathrm{eV}^{-1}$ and $7.57 \times 10^{12} \mathrm{~cm}^{-2} \mathrm{eV}^{-1}$, respectively. Hence, annealing process reduces the number of interface traps considerably. This, in turn, would make more carriers available for the conduction process. 

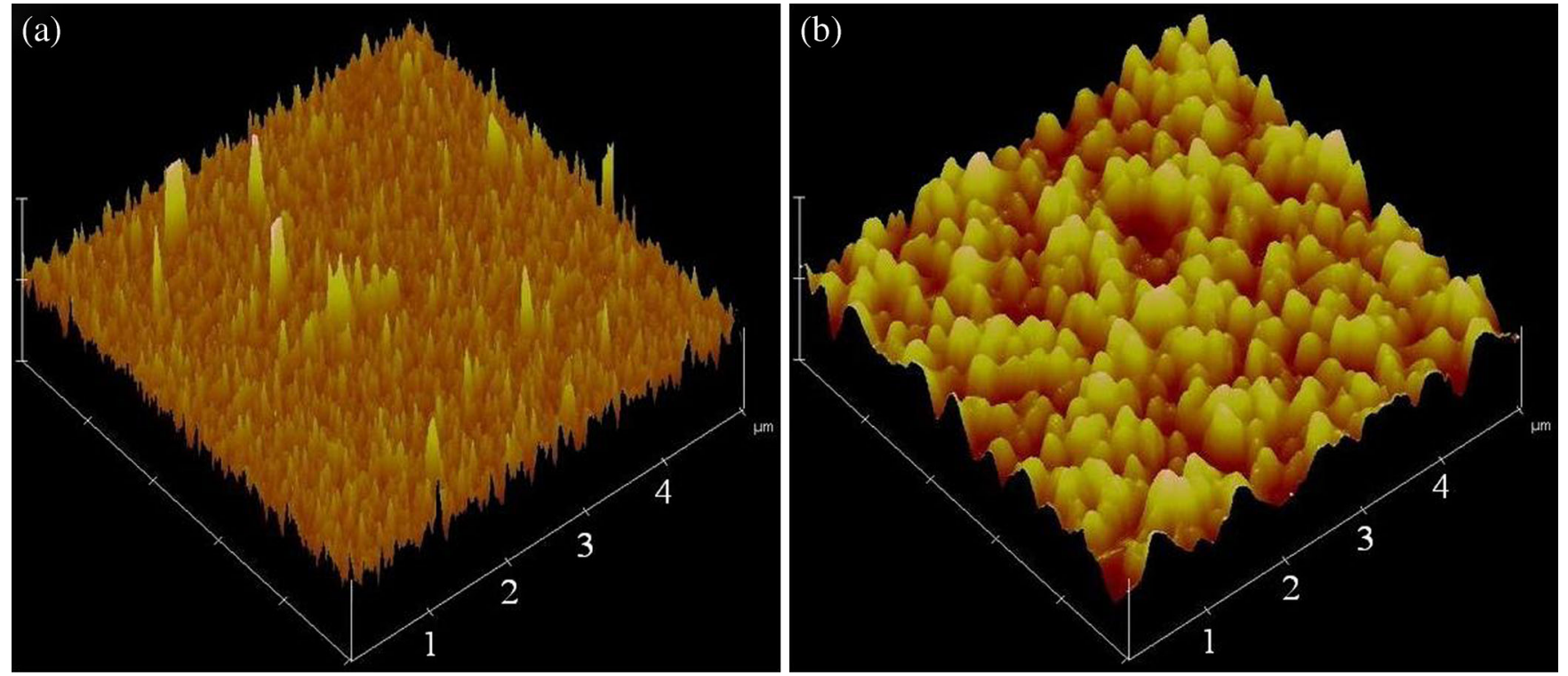

Figure 7. Morphology of $\mathrm{ZnPc}$ film grown over parylene: (a) as-deposited and (b) annealed at $120{ }^{\circ} \mathrm{C}$ for $3 \mathrm{~h}$.

Figure 6 shows a typical AFM topograph of $450 \mathrm{~nm}$ thick parylene film. The root-mean square (rms) surface roughness of the film is $\sim 7.9 \mathrm{~nm}$ over an area of $2 \times 2 \mu \mathrm{m}^{2}$.

Figure 7 shows morphology of the as-deposited and annealed $\mathrm{ZnPc}$ over parylene films. On comparison of these films, we can see larger grains in the annealed samples, which are responsible for their superior electrical properties. It is well established that annealing increases the crystallinity in phthalocyanine thin films. An improvement in crystallinity is observed in CuPc on annealing (Yanagiya 2003). Recently, in vacuum evaporated films of zinc octakis octyloxy phthalocyanine $\left(\mathrm{ZnPcOC}_{8}\right)$, it is found that crystallinity increases with increase in annealing temperature (Vinu and Menon 2012). The most plausible explanation for the improved performance of OFETs is, thus, the improvement in their crystallinity and the consequent effective carrier transport through the active layer.

\section{Conclusions}

Organic field effect transistors were realized using zinc phthalocyanine as the organic active layer and parylene as the organic gate. The measurement of the electrical characteristics of the as-deposited and annealed samples was carried out at room temperature. It was found that the annealed samples exhibit remarkably improved transistor characteristics. The post deposition annealing also improves the crystallinity of the organic layer and in turn the mobility of the device. We also observed a considerable reduction in the number of interface traps for the annealed devices. The mobility observed in this transistor configuration for $\mathrm{ZnPc}$ is the highest among the various phthalocyanines. In general, the post- deposition annealing enhances the transistor characteristics of OFETs based on $\mathrm{ZnPc}$.

\section{Acknowledgement}

This work was supported by the Millimeter-Wave Innovation Technology Research Centre (MINT), Dongguk University, Republic of Korea.

\section{References}

Belgachi A and Collins R A 1998 J. Phys. D: Appl. Phys. 211647 Brütting W 2005 Physics of Organic Semiconductors, (Wiley-VCH: Weinheim) Chapter 1

Chua L-L, Zaumseil J, Chang J-F, Ou E C-W, Ho P K-H, Sirringhaus H and Friend H 2005 Nature 434194

Dimitrakopoulos C D and Malenfant P R L 2002 Adv. Mater. 1499

Fang S, Kohama K, Hoshi H and Maruyama Y 1993 Jpn. J. Appl. Phys. 32 L1418

Gershenson M E, Podzorov V and Morpurgo A F 2006 Rev. Mod. Phys. 78973

Gould R D 1985 Thin Solid Films 12563

Halik A M, Klauk H, Zschieschang U, Schmid G, Radlik W and Weber W 2002 Adv. Mater. 141717

Horowitz G 1998 Adv. Mater. 10365

Knipp D, Muck T, Benor A and Wagner V 2006 J. Non-Cryst. Solids 3521774

Liqiang Li, Qingxin Tang, Hongxiang Li, Wenping Hu, Xiaodi Yang, Zhigang Shuai, Yunqi Liu and Daoben Zhu 2008 Pure. Appl. Chem. 802231

Ling M-M, Bao Z and Li D 2006 Appl. Phys. Lett. 88033502

Meijer E J, Tanase C, Blom $\mathrm{P}$ W M, van Veenendaal E, Huisman B-H, de Leeuw D M and Klapwijk T M 2002 Appl. Phys. Lett. 803838 
Pernstich K P, Oberhorff D, Goldmann C and Batlogg B 2005 Appl. Phys. Lett. 89213509

Podzorov V, Pudalov V M and Gershenson M E 2003 Appl. Phys. Lett. 821739

Rolland A, Richard J, Kleider J-P and Mencaraglia D $1993 \mathrm{~J}$. Electrochem. Soc. 1403679

Sundar V C, Zaumseil J, Podzorov V, Menard E, Willett R L, Someya T, Gershenson M E and Rogers J A 2004 Science 303 1644
Tsetseris L and Pantelides S T 2007 Phys. Rev. B75 153202

Unni K N N, Sylvie Dabos-Seignon and Nunzi Jean-Michel $2005 \mathrm{~J}$. Phys. D: Appl. Phys. 381148

Veres J, Ogier S and Lloyd G 2004 Chem. Mater. 164543

Vinu T V and Menon C S 2012 J. Nano-Electron. Phys. 4 04005

Wang H, Zhu F, Yang J, Geng Y and Yan D 2007 Adv. Mater. 19 2168

Yanagiya S 2003 J. Crystal Growth 254244 